\title{
Erratum to: The Breast and Oncoplastic Multidisciplinary Team
}

Fiona MacNeill, Marios Konstantinos Tasoulis, Melissa Ley Hui Tan, and Andreas Karakatsanis

\section{Erratum to}

L. Wyld et al. (eds.), Breast Cancer Management for Surgeons, https://doi.org/10.1007/9783-319-56673-3_16

In the original version of chapter 16, the author Andreas Karakatsanis was missed. 